\title{
A Study of Power Saving TechniQue IN WIRELESS NETWORKS
}

\author{
Mamta Agiwal $^{1}$ and Kashif Ahmed ${ }^{2}$ \\ ${ }^{1}$ Department of Electrical and Electronics Engineering, CMRIT, Bangalore, \\ India \\ mamta_wadhwa@hotmail.com \\ ${ }^{2}$ Department of Electrical and Electronics Engineering, CMRIT, Bangalore, \\ India \\ kashifahmedy2k10@gmail.com
}

\begin{abstract}
Much research on wireless networks have focused on the power consumption of the wireless nodes, while at the same time how to acquire power from ambient environment is another direction to extend the battery lifetime. Though, mostly extending the lifetime of WSNs rely on making the electronic circuitry power efficient by incorporating advances in node architecture, transceivers, access protocols and on finite energy sources like batteries. In contrast, WSNs Powered by Ambient Energy Harvesting can also prove to be useful and economical in the longterm as they can operate for very long periods of time until hardware failure, because ambient energy can be harvested from the environment perpetually. Although cellular networks account for a rather small share of energy use, lowering their energy consumption appears beneficial from an economical perspective. In the strive for lessening of the environmental impact of the information and communication industry, energy consumption of communication networks has recently received increased attention. The paper discusses the various techniques for increasing the life of WSNs.
\end{abstract}

\section{KEYWORDS}

DVS, Sensor Node Architecture, Medium Access Protocols, Ultracapacitors, RF Transceivers

\section{INTRODUCTION}

Traditionally, systems were wire based, which were essentially extensions of laboratory based Data Acquisition (DAQ) Systems. To overcome the many disadvantages of wired systems, use of wireless technologies have been proposed. They can be cellular networks or WSN. Cellular networks are widely used for mobile communication whereas Wireless Sensor Networks (WSNs) leads to different applications that involve a tight coupling between computing infrastructure and the physical world.

A Cellular network is one of the radio network distributed over land areas called cells, each served by at least one fixed-location transceiver known as a cell site or base station. When these cells joined together provide radio coverage over a wide geographic areas. Cellular networks provides the advantages such as increased capacity, reduced power use, large coverage area, reduced interference from other signals. In cellular architecture the network is partitioned into a virtual grid of cells to perform fault detection and recovery locally with minimum energy consumption.

Sundarapandian et al. (Eds): CoNeCo,WiMo, NLP, CRYPSIS, ICAIT, ICDIP, ITCSE, CS \& IT 07, pp. 341-351, 2012. (C) CS \& IT-CSCP 2012

DOI : $10.5121 /$ csit.2012.2433 
The general applications of wireless sensor networks require many wireless sensor nodes to form a network to sense the signal of interest. Extreme events like earthquakes can cause enormous damage to civil infrastructures without producing any apparent visible change. Such damage can result in life threatening conditions evolving in the structure either in the immediate aftermath or long after the actual event has happened. Near real-time structural monitoring of civil infrastructure reduces the loss of human lives by warning of hazardous structures and impending collapses and also provides information to emergency response services. Moreover, the infrastructure may undergo gradual deterioration over the time due to different environmental, physical and chemical factors.

However the common problem in both the networks is the source of energy which is most often an attached battery cell. Thus, a wireless system throws up the new problems being that of power. Due to the limited capacity of batteries and the difficulty of frequent battery recharging or replacement, energy is a scarce and precious resource. Decreasing the energy consumed during system operation, will directly translates to increased lifetime

This article discusses several such emerging techniques that will help achieve the goal of longlasting wireless networks. In these paper techniques for energy-efficient architecture is presented along with DVS techniques for power management, followed by a discussion of ultra-low power medium access protocols and RF Transceiver design.

The techniques for environmental energy harvesting are described. The articles also discuss the use of super capacitors instead of batteries as energy storage devices.

\section{ENERGY EFFiCIENT ARCHITECTURE}

\subsection{Energy Aware Cooperative Base Station}

A base station is the interface between wireless phones and traditional wired phones. The base station, a wireless system, uses microwave radio communication. It is composed of several antennas mounted on a tower and a building with electronics in it at the base. When a call is made using cell phone, the cell phone and base station communicate back and forth by radio, and the radio waves they use are in the microwave region of the electromagnetic spectrum.

Traffic load at the base station has significant variations in time due to a number of factors like user mobility and behaviour. Compared to the residential areas the traffic load is higher in industrial areas during daytime, and vice versa during the night. As a result, there are some cells which are always under low load, while some are under heavy traffic load. Hence, a static cell size deployment is not optimal with varying traffic conditions.

Since BS consumes considerable amount of energy, selectively letting BSs go to sleep based on their traffic load can lead to significant amount of energy savings. When some cells are switched off or in sleep mode, the radio coverage can be guaranteed by the remaining active cells by filling in the gaps created. The heterogeneous multiprocessors technique discussed for sensor node platform can also be implemented at the BS.

\subsection{Sensor Node Platform}

The most crucial aspect of sensor network energy optimization is the design of the sensor-node platform itself. Ultimately, it is the sensor-node hardware that consumes the energy, so if the platform itself is not energy-efficient, no amount of higher layer optimization will yield desired results. Before we discuss energy efficient design techniques for sensor-node platforms, let us look at the typical workload profile for a sensor node that is part of a surveillance sensor network. This is shown in Fig. 1. 
As can be seen, the workload consists of two distinct phases. The first phase, labelled "low workload," represents the state of the network in the absence of intruders. Nodes periodically wake up, sample their sensors to detect any intruders, and, in their absence, go back to sleep. To provide high energy efficiency in this phase, a sensor-node platform should:

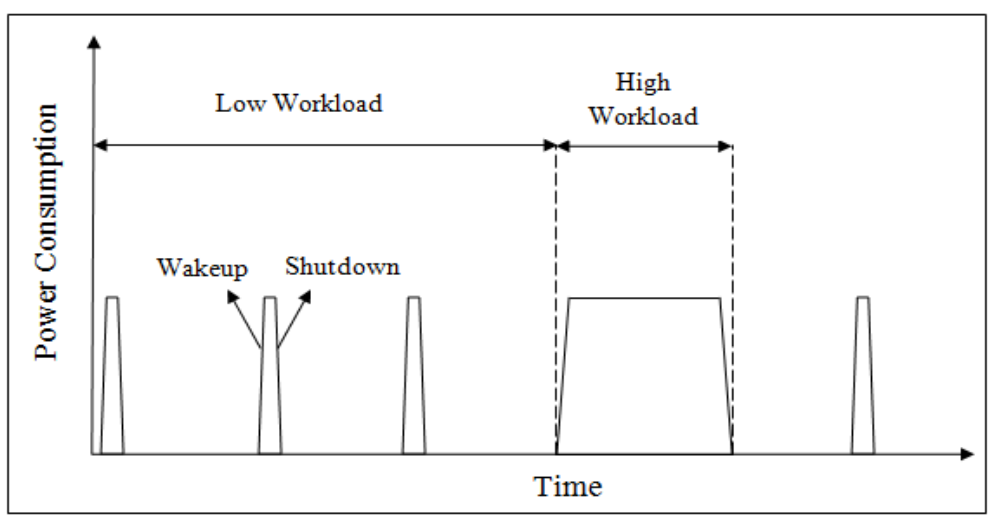

Fig. 1 Typical wireless sensor network workload showing to distinct phases

- Provide an ultra-low power sleep mode since the node spends a majority of its time in sleep state

- Provide a rapid wakeup capability to minimize the power management (duty-cycling) overhead.

The second phase of the sensor network's workload is labelled "high workload" in Fig. 1. This represents the state when intruder activity is detected. In this state, the nodes perform a significant amount of computation and communication to locate and track the intruder. To optimize energy in this phase, the sensor node should perform its active-mode computation and communication in an energy-efficient manner.

Existing sensor-node platforms are unable to simultaneously satisfy all the above requirements. While an ultra-low-power sleep mode can be achieved through techniques such as power gating, negligible power-management overhead and high active mode energy-efficiency represent conflicting objectives. This reflects the basic trade-off that processors with low power management overhead have poor active-mode energy efficiency and vice-versa. One way to overcome this conflict is to design a sensor node with both types of processors, as shown in Fig. 2.

In the "low workload" phase, the high-end processor is power gated (i.e., completely powered off) and the sensor node uses a low-end processor that provides low overhead wakeup. When the sensors detect activity, the network transitions to the "high workload" phase. The high-end processor is woken up and performs the intensive computation and communication in an energyefficient manner.

Once the intruder leaves, the network transitions to the "low workload" phase again and the highend processor is shut down. Such a tiered processing architecture is called a heterogeneous multiprocessor with staged wakeup. Unlike conventional multiprocessor systems where the processors usually operate in parallel, in this case, only one of the two processors is used to execute application functionality at any given time. The challenge then is to decide when and how to switch from one processor to the other at runtime based on the observed workload. 


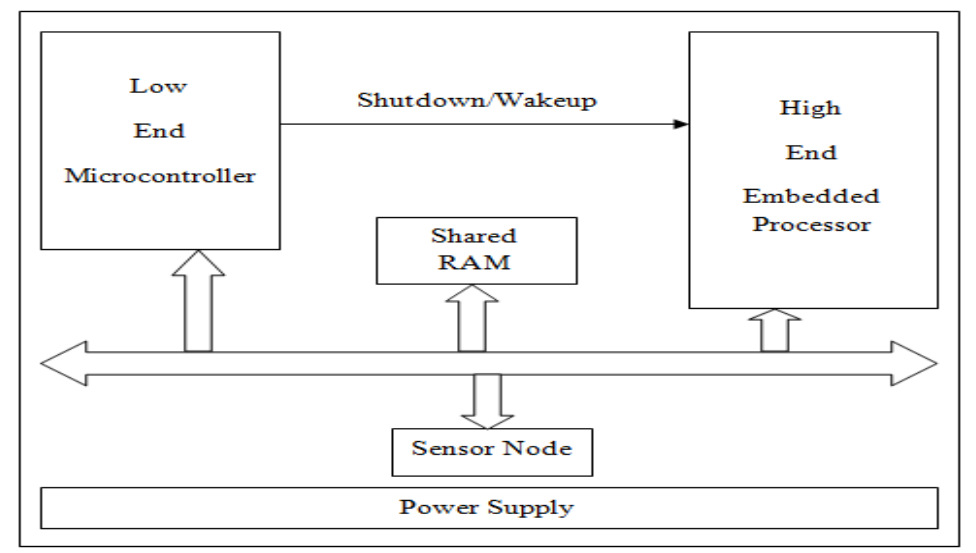

Fig. 2 A heterogeneous multiprocessor sensor node with staged wakeup

\section{Dynamic Voltage Scaling}

While shutdown techniques can yield substantial energy savings in idle system states, additional energy savings are possible by optimizing the sensor node performance in the active state. Dynamic voltage scaling (DVS) is an effective technique for reducing CPU (central processing unit) energy.

Most microprocessor systems are characterized by a time-varying computational load. Simply reducing the operating frequency during periods of reduced activity results in linear decreases in power consumption but does not affect the total energy consumed per task. Reducing the operating voltage implies greater critical path delays, which in turn compromises peak performance. Significant energy benefits can be achieved by recognizing that peak performance is not always required and therefore the processor's operating voltage and frequency can be dynamically adapted based on instantaneous processing requirement.

The goal of DVS is to adapt the power supply and operating frequency to match the workload so the visible performance loss is negligible. The crux of the problem is that future workloads are often nondeterministic.

The rate at which DVS is done also has a significant bearing on performance and energy. A low update rate implies greater workload averaging, which results in lower energy. The update energy and performance cost is also amortized over a longer time frame. On the other hand, a low update rate also implies a greater performance hit since the system will not respond to a sudden increase in workload. The DVS power saving technique can be effective for both wireless sensor networks as well as cellular networks.

\section{Design of Ultra-Low Power Medium Access Protocols}

\subsection{Power Saving Protocols in Cellular Networks}

In the current cellular network architecture based on WCDMA/HSPA, BSs and mobile terminals are required to continuously transmit pilot signals. Newer standards such as LTE, LTE-Advanced and WiMAX have evolved to cater ever-growing high speed data traffic requirements. With such high data requirements, although BSs and mobile units (MU) employing newer hardware (such as 6 multiple-input and multiple-output (MIMO) antennas) increase spectral efficiency allowing to transmit more data with the same power, power consumption is still a significant issue for future 
high speed data networks and they require energy conservation both in the hardware circuitry and protocols. The MAC layer protocol discussed for sensor networks can also be applied to cellular networks.

\subsection{MAC Layer Protocol in Sensor Networks}

Radio duty cycling, where the radio is off by default but wakes up periodically to participate in potential network communication, has received significant attention in the literature. However, the duty-cycling benefits achieved in theory and simulations have often not translated to practice. This can be attributed mainly to the problem of time uncertainty between sensor nodes, which is illustrated in Fig. 3(a).

If the sleep/wakeup schedules of nodes do not overlap, they cannot communicate with each other. Note that each sensor node has its own notion of time governed by its local clock. The approaches used by MAC protocols to address this problem of time uncertainty determine their energy consumption.

Existing MAC layer protocols can be broadly classified into being either asynchronous or synchronous approaches. As the name suggests, asynchronous protocols do not rely on any relative time-synchronization information between the two communicating nodes. Instead, each packet is transmitted with a long enough preamble so that the receiver is guaranteed to wake up during the preamble transmission time, as shown in Fig. 3(b).

This implies that the preamble transmission time (preamble size divided by the radio's data rate) should be at least equal to the duty-cycling period which clearly indicates the significant energy overheads. Further, using a long preamble for every packet decreases the effective channel capacity and also increases the receiving and listening overhead. All the overhearing nodes will have to be awake for half of the preamble transmission time, on average, before receiving the destination address information in the packet header and going back to sleep if they are not the intended receivers. A wakeup-frame-based scheme tries to reduce this overhead by transmitting multiple small wakeup frames instead of a single long preamble before the payload, as shown in Fig. 3(c).

Each wakeup frame includes the destination address, so that overhearing nodes can go to sleep just after receiving a single wakeup frame. Furthermore, each wakeup frame also has a sequence number so that the destination node can calculate the start time of the data frame, after receiving the wakeup frame. Using this information, the receiver can decide to go back to sleep for the time interval, equal to the transmission time of the rest of the wakeup frames.

On the other hand, synchronous approaches try to maintain time-synchronized duty cycling of nodes. This enables the transmitter to turn on the radio at the right moment, and transmit the packet without incurring any extra overhead of a longer preamble or multiple wake-up frames, as shown in Fig. 3(d). However, time synchronization is achieved by periodically exchanging control beacons, which results in a significant energy overhead, especially for low-data rate applications.

Instead of being completely asynchronous or synchronous, an alternative is an uncertainty driven approach to duty cycling, where a model of long-term clock drift is used to minimize the dutycycling overhead. At the time of transmitting a packet, the node predicts its relative drift with the receiver node and chooses the preamble size accordingly instead of always choosing the worstcase preamble size. Further, instead of keeping the nodes in perfect synchrony at all times, just the right amount of synchronization is maintained between the nodes that minimizes the total 
overhead of achieving the synchronization precision and the extra preamble bytes that are added to every packet to overcome the time uncertainty.

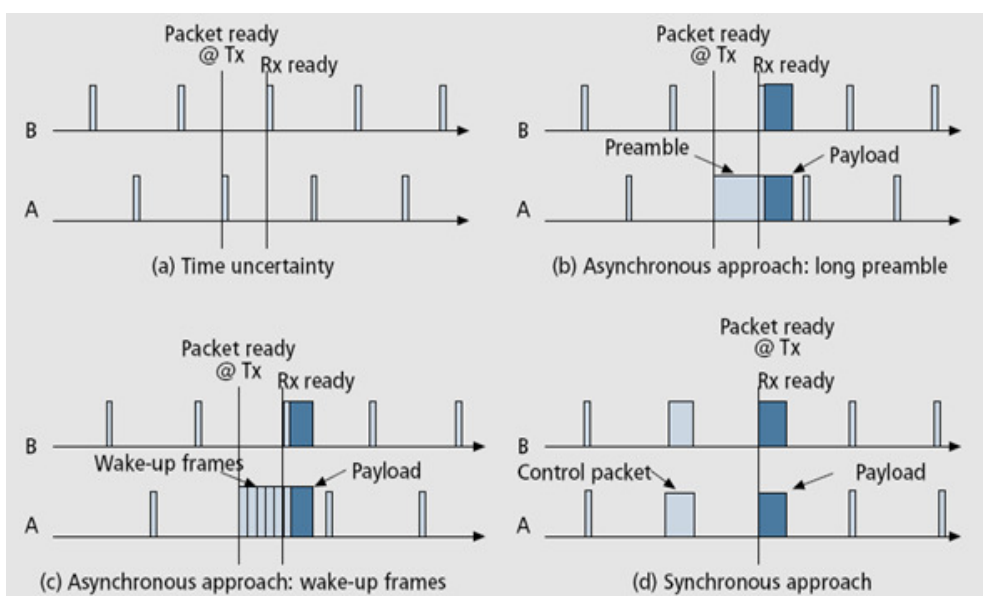

Fig. 3 commonly used approaches for medium access control in sensor networks

\section{RF TRANSCEIVER DeSIGN ON SENSOR NODE}

\subsection{Improvements in Power Amplifier at Base Station}

There are three essential parts of a base station: radio, baseband and feeder. Out of these three, radio consumes more than $80 \%$ of a base station's energy requirement, of which power amplifier (PA) consumes almost 50\%. Shockingly, $80-90 \%$ of that is wasted as heat in the PA, and which in turn requires air-conditioners, adding even more to the energy costs.

To obtain high linearity of the PAs in order to maintain the quality of radio signals, PAs have to operate well below saturation, resulting in poor power efficiency PAs based on special architectures such as digital pre-distorted Doherty-architectures and GaN (Aluminium Gallium nitride) based amplifiers seem to be more promising by pushing the power efficiency levels to over $50 \%$.

Since GaN structures can work under higher temperature and higher voltage, they can potentially provide a higher power output. Additional improvements in efficiency can be obtained by shifting to switch-mode PAs from the traditional analog RF-amplifiers. Compared to standard analog PAs, switch-mode PAs tend to run cooler and draw less current.

\subsection{RF Transceivers for Wireless Sensor Network}

Operating RF transceiver is a key to save power since RF transceiver is the most powerconsuming part of a wireless sensor node. Obviously, reducing the RF output power will reduce the power consumption in the same transmission time, but it also makes the transmission range shorter. Because the sensor node connects to the local control centres (LCC) directly in our network topology, shorter transmission range means smaller coverage of a sub-network.

In addition, smaller coverage of a sub-network also means that we will need to divide the monitoring area into more sub-networks and need to have more LCCs consequently. One thing that should be taken into account is that the coverage of each sub-network will inevitably have some overlap; therefore, we will need to schedule the operating time of sub-networks that are adjacent to each other. 
If the number of sub-networks is larger, it will be more complicate to coordinate each LCC's operation. On the other hand, the number of sensor nodes is another parameter that should consider deciding the number of LCCs. We usually do not let a sub-network contain too much sensor nodes since it will make the minimum interval of a sensor node reporting the measuring result to the LCC too long, which may make the transmission delay become unacceptable.

In short, the decision of the transmission range should consider many aspects, including power consumption, required coverage, complication of scheduling problem, and the number of sensor nodes. Because there are a lot of inferring sources and obstacles that will cause interference to the wireless communication, sensor nodes that have the same distance to the LCC do not have the same received signal strength. Adjusting the RF output power according to the signal to noise ratio ( $\mathrm{S} / \mathrm{N}$ ratio) is a way to save power and to ensure good communication quality at the same time. Because the RF output power of this RF transceiver is also programming adjustable, we can adjust the RF output power dynamically by the program. It will not only save more power, but also can ensure good S/N ratio on every sensor node and can reduce the overlap of different subnetwork.

However, if the distance between the farthest and nearest sensor node is short, it is not necessary to adjust the RF output power dynamically since the power saved by this method is not significant in this situation. Besides RF output power, the data rate of transmission is another factor that will affect the power consumption. Intuitively, higher data rate can have lower transmission time for the same amount of data and thus can have lower power consumption.

However, the relationship between power consumption of RF transceiver and the data rate is not simply inverse proportional. The reason comes from the fact that when the data rate is higher, the sensitivity of the RF receiver will be lower. In other words, if the RF output power remains the same, the coverage of a sub-network will be smaller when the data rate is higher. Saying it in another way, we can use lower RF output power to meet the same requirement of transmission range when the data rate is lower.

One thing to keep in mind is that although higher data rate seems more power-efficient, transmitting data at higher data rate will make the maximum transmission range shorter due to lower receiver sensitivity.

\section{Beyond Power Management: Environmental Energy HARVESTING}

In several remote locations of the world such as Africa and Northern Canada, electrical grids are not available or are unreliable. Cellular network operators in these off-grid sites constantly rely on diesel powered generators to run base stations which is not only expensive, but also generates $\mathrm{CO} 2$ emissions. Moreover, this fuel has to be physically brought to the site and sometimes it is even transported by helicopter in remote places, which adds further to this cost. In such places, renewable energy resources such as sustainable bio fuels, solar and wind energy seem to be more viable options to reduce the overall network expenditure. Hence, adopting renewable energy resources could save cellular companies such recurrent costs, since they are capital intensive and cheaper to maintain. Also, since renewable energy is derived from resources that are regenerative, renewable energy resources do not generate greenhouse gases such as $\mathrm{CO} 2$. The new approaches using energy harvesting from the environment can be a promising technique to increase the system performance in terms of energy efficiency in future wireless mobile networks

An emerging technique that promises to circumvent this limitation is environmental energy harvesting. Ongoing power management developments enable battery-powered electronics to live 
longer. Such advances include Energy efficient sensor node architecture, dynamic power management using DVS techniques, RF transceiver design and clever wake-up procedures. Exploiting renewable energy resources in the device's environment, however, offers a power source limited by the device's physical survival rather than an adjunct energy store.

Energy harvesting's true legacy dates to the water wheel and windmill, and credible approaches that scavenge energy from waste heat or vibration have been around for many decades. Nonetheless, the field has encountered renewed interest as low-power electronics, wireless standards, and miniaturization conspire to populate the world with sensor networks and mobile devices. Energy from the environment can be tapped in various forms as discussed.

\subsubsection{Energy From Maxwell}

With copious radio transmitters scattered throughout today's urban landscape, we might consider background radio signals as a mobile-device power reservoir. Electronic systems that harvest energy from ambient-radiation sources, however, have extremely limited power and generally require either a large collection area or close proximity to the radiating source.

Although crystal radios derive their energy from broadcast signals, the receiving antenna can be restrictively large unless the set is close to a transmitter, and access to a good ground is usually required. Even so, a crystal set's energy harvest is limited, typically on the order of tens of microwatts.

Another approach, heralding Nicola Tesla a century ago and William C. Brown 50 years later, involves broadcasting RF energy deliberately to power remote devices. This practice is now commonplace in passive radio-frequency identification systems, which derive their energy inductively, capacitively, or radiatively from the tag reader.

Ambient light presents another opportunity to scavenge power. The energy conversion efficiency of relatively inexpensive crystalline silicon solar cell modules is generally below 20 percent and closer to 10 percent for flexible amorphous silicon panels (standard solar cells produce roughly $100 \mathrm{~mW} / \mathrm{cm}^{2}$ in bright sun and $100 \mu \mathrm{W} / \mathrm{cm}^{2}$ in a typically illuminated office).

Accordingly, the lack of strong, consistent sunlight constrains applications. Nonetheless, the established products that harness light as an energy source span several orders of magnitude in power. These range from solar homes producing kilowatts on a bright day, to solar battery chargers for cell phones that purport to produce up to $2 \mathrm{~W}$ of power in direct sunlight, to a PDA that runs off a panel of solar cells lining.

\subsubsection{Thermoelectric Conversion}

Objects or environments at different temperatures offer the opportunity for energy scavenging via heat transfer (the heat engine running in a geothermal power station is a familiar large-scale example).

The Carnot cycle-comprising adiabatic and isothermal operations - provides the fundamental limit to the energy obtained from a temperature difference. Mechanical products have been designed to run off the meagre energy they harvest from ambient thermal cycling.

\subsubsection{Vibrational Excitation}

From the subtle vibrations of floors and walls that nearby machinery causes to the severe excitation of an automobile chassis or jet engine housing, mechanical stimuli are common in 
many settings but can vary widely in frequency and amplitude. Inventors have long designed systems to harvest this energy, usually by exploiting the oscillation of a proof mass resonantly tuned to the environment's dominant mechanical frequency. Self-winding watches use the motion of the user's body to wind their mechanisms.

Abraham-Louis Perrelet created the first known self-winding pedometer watch around 1770, Widespread adoption of these systems didn't occur until after the 1930s, when watch cases could be hermetically sealed to protect the mechanism from dust. A modern self-winding wristwatch contains an approximately 2 -gram rotary proof mass mounted off-centre on a spindle. As the user moves during the day, the mass spins and winds the mechanism.

Most sensors used in WSNs today rely on limited and non-renewable energy sources like batteries to operate. Advances in ambient energy harvesting technologies based on energy from Radiations, thermoelectric conversions and vibration excitation can be incorporated with the sensor nodes to harvest energy from environment. Since batteries have limited recharge cycles, super capacitors with virtually unlimited recharge cycles are an attractive option for use in such WSNs to replace batteries because they can operate perpetually without the need for replacement. Some examples have been deployed in test beds.

\section{SUPERCAPACITOR OR ULTRACAPACITOR}

A supercapacitor or ultracapacitor is an electrochemical capacitor that has an unusually high energy density when compared to common capacitors. Ultra capacitor provides high energy density and exhibits low equivalent series resistance (ESR).

The combination of low ESR and extremely low inductance provides the Ultracapacitor with a very high power density and fast rise time as well. As a double layer capacitor, the Ultracapacitor is not constrained by the same limitations as dielectric capacitors. Thus, although its discharge characteristics and equivalent circuit are similar to dielectric capacitors. the capacitance of the Ultracapacitor increases with the ceramic loading on the substrate and its ESR is inversely proportional to the cross-sectional area of the device.

The Ultracapacitor principle has been extended to non-aqueous electrolytes.

\subsubsection{Advantages of Ultracapacitor}

- high expected lifetime, more than 10 years

- ultracapacitors have high power density

- short charge-discharge time

- maintenance free

- wide operating temperature

- less weight

- short circuit protection

\subsubsection{Design of Ultracapacitor}

- at every interface, an array of charged particles and oriented dipoles is thought to exist

- this array is known as an electrical double layer

- the capacitance observed in electrical double layer capacitors arises from the charge stored at the interface by an externally imposed electric field 


\subsubsection{WSNs Using Ultracapacitor}

Use supercapacitors instead of batteries as energy storage devices, providing virtually unlimited recharge cycles for perpetual deployment. However, as the rate of charging is usually much lower than the rate of energy consumption for the sensor nodes, sensor network can only be awake for a short period of time before it needs to shut down in order to recharge. Moreover, the time taken to charge up the sensor varies due to environmental factors. Fig. 5 shows the salient difference in the energy models of batteries versus energy harvesting devices.

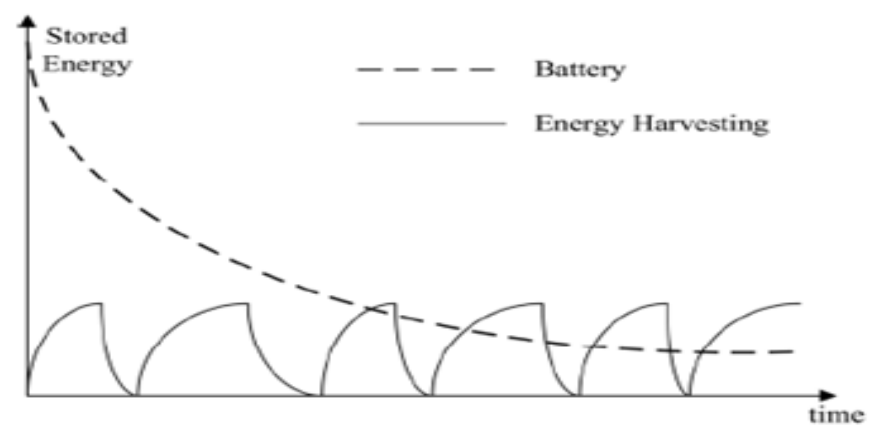

Fig. 4 Characteristics of energy sources

\subsubsection{WSNs Using Ultracapacitor}

- they can operate for long periods of time until hardware failure

- they use supercapacitors instead of batteries as energy storage device

- they provide virtually unlimited recharge cycles for perpetual deployment

- the rate of charging is usually much lower than the rate of energy consumption for the sensor nodes

\section{Conclusions}

In WSNs and cellular networks power is premium and important to increase longitivity of the sensor nodes. Understanding of various power saving techniques, along with power harvesting methods can help enhance the lifetime of sensor nodes. The upgrading of architecture, power management methods, low power protocols and environmental energy harvesting if applied to WSNs and cellular will increase their performance for extended period of time.

\section{REFERENCES}

[1] Yi Shi, and Y. Thomas Hou, "Some Fundamental Results on Base Station Movement Problem for Wireless Sensor Networks" IEEE/ACM transactions on networking, vol 20, no.4 pp. $1054-1067$, Aug 2012

[2] Jayashree V. Shiral and Bhushan N. Mahajan, "A Review on- Comparative Study of Issues in Cellular, Sensor and Adhoc Networks" David C. Wyld, et al. (Eds): CCSEA, SEA, CLOUD, DKMP, CS \& IT 05, pp. 199-205, 2012

[3] C. Han et al., "Green radio: radio techniques to enable energy-efficient wireless networks," IEEE IEEE Commun. Mag., vol. 49, no. 6, pp. 46-54, 2011

[4] Ziaul Hasan, Hamidreza Boostanimehr, and Vijay K. Bhargava, "Green Cellular Networks: A Survey, Some Research Issues and Challenges"CS.NI 24 Sep 2011

[5] I. Humar, X. Ge, L. Xiang, M. Jo, M. Chen, and J. Zhang, "Rethinking energy efficiency models of cellular networks with embodied energy," IEEE Network Magazine, vol. 25, no. 2, pp. 40-49, 2011 
[6] Fred Richter and Gerhard Fettweis, "Cellular Mobile Network Densification Utilizing Micro Base Stations" Communications (ICC), 2010 IEEE International Conference, pp. 1-6, 23-27 May 2010.

[7] L. M Correia, et. al, "Challenges and enabling technologies for energy aware mobile radio networks," IEEE Communications Magazine, vol.48, no.11, pp.66-72, November 2010

[8] Simjee F. I., and Chou P. H., "Efficient Charging of Supercapacitors for Extended Lifetime of Wireless Sensor Nodes",in IEEE Transactions on Power Electronics,Vol. 23, No. 3, May 2008, pp. 1526-1536

[9] Vijay Raghunathan, Saurabh Ganeriwal, and Mani Srivastava, UCLA, "Emerging Techniques for Long Lived Wireless Sensor Networks”, IEEE Communications Magazine, pp. 108-114, April, 2006

[10] X. Jiang, J. Polastre, and D. Culler, "Perpetual Environmentally Powered Sensor Networks", IEEE International Symposium Info Process. Sensor Networks, 2005, pp. 463-468

[11] Joseph A. Paradiso and Thad Starner, "Energy Scavenging for Mobile and Wireless Electronics", published by the IEEE CS and IEEE ComSoc, Pervasive Computing, January-March 2005, pp. 19-27

[12] C. Enz et al. "WiseNET: An Ultra Low Power Wireless Sensor Network Solution", IEEE Computer,Vol. 37, No. 8, August 2004, pp. 62-70

[13] Rahimi M., Shah H., Sukhatme G. S., Heidemann J., and Estrin D., "Studying The Feasibility of Energy Harvesting in a Mobile Snsor Network", in proceeding of the IEEE International Conference on Robotics and Automation(ICRA), Sep 2003, pp.19-24, Taipei, Taiwan

[14] J. M. Rabaey, M. J. Ammer, J. L. Silva Jr, D. Patel and S. Roundy, "PicoRadio Supports Ad Hoc Ultra-Low Power Wireless Networking,” IEEE Computer, Vol. 33,pp. 42-48, July, 2000

[15] K. Sohrabi, J. Gao, V. Ailawadhi and G. J. Pottie, "Protocols For Self-organization of a Wireless Sensor Network," IEEE Personal Communications, Vol. 7(5),pp. 16-27, 2000

[16] Wen-Jong Wu, Yi-Fan Chen, Yu-Yin Chen, Chao-Sheng Wang and Yin-Shen Chen, Smart Wireless Sensor Network Powered By Random Ambient Vibration

[17] IEEE 802.15.4-2006 Stds, Wireless Medium Access Control (MAC) and Physical Layer (PHY) Specification for Low-Rate Wireless Personal Area Networks(WPANs)

[18] Zhi Ang Eu, Winston K. G. Seah, and Hwee-Pink Tan "A Study of MAC Schemes for Wireless Sensor Networks Powered By Ambient Energy Harvesting”

\section{Authors}

Mamta Agiwal is working as an Associate Professor in Dept. of EEE at CMR Institute of Technology, Bangalore. She is pursuing Ph.D. at Jain University, Bangalore. She has done her B.Tech in Electrical and Electronics Engineering and M.Tech in Digital Electronics. Her research interests are Wireless Networks.

Kashif Ahmed is working as an Assistant Professor in Dept. of EEE at CMR Institute of Technology, Bangalore. He is pursuing M.Tech in VLSI and is B.E in Electrical and Electronics Engineering. His research interest is Wireless Networks.

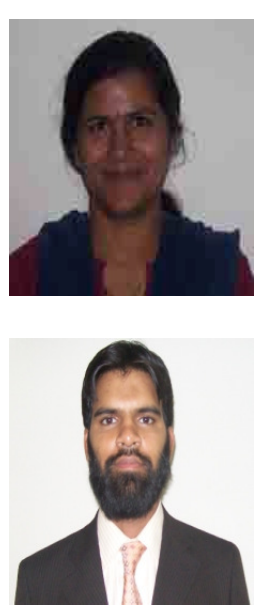

